\title{
HACER MATEMATICAS
}

\author{
FRANCISCO HERNÁN (del GRUPO CERO de Vaiencia)
}

\begin{abstract}
SUMMARY
Reproductive thinking, the habit of using ready-made answers, has two advantages: it is easier both to reach and to test. Productive thinking, creating new answers and using new organizations is more difficult to teach and needs more time and more dynamic teaching relations.

Through the process of two problem-solving situations fone by 14-15 years old pupils and the other by $15-16$ years old) some reasons are suggested in this paper which argue in favour of mathematical activities more creative than repetitive.
\end{abstract}

\section{INTRODUCCION}

Una peridista acababa de llegar a casa de Gabriel Garcia Márquez para despertarlo con la nueva de que ya cra Premio Nóbel. Enhorabuena, icómo se siente?, ¿está Irabajando en algo aciualmente? «Pues si. Estoy escribiendo una novela en la que la gente es feliz. Creo que hay que hacer algo para que la felicidad esté más de moda".

¿Quièn no esperará com impaciencia una novela que nace de idea tan bonita? Cabe imaginar paralelamente que con ese talento Garcia Márquez no estará lejos de sentir, si no impaciencia, al menos ajguna solidaridad con aquellos que quieran escribir sobre el éxito escolar. A ver si se pone de moda.

Son muchos los que están de acuerdo en que en la ensehanza de las matemáticas se alcanza algún éxito cuando se ven realizados lodos - o por lo menos algunosde los deseos siguientes:

Presentar las matemáticas como una materia para usar y para disfrutar. Las matemáticas, como tantas otras cosas, deben proponerse que quien las practica supere un umbral minimo de satisfacción. Esa satisfacción puede discurrir por tres canales: el del placer, el del conocimiento, el del uso. Cuando no se alcanza ese umoral minimo se ha perdido miserablemente el tiempo.

Promover una actitud favorable hacia ellas. Lo cual beneficiará sin duda al profesor igualmente. Para los profesores de alumnos insatisfechos, la expriencia es. colar es también generaimente insatisfactoria.

Desarrollar la confianza en su uso. Evitando el rectiazo a algo que está fuera de los límites de la comprensión y del éxito, y el amargo sabor de derrota con que gran número de alumnos sale del colegio o del Instituto.
Favorecer el movimiento de puesta en marcha. Comprender la solución de un problema que otra persona ha resuelto es algo muy simple a veces; pero generalmente es mucho más dificil descubrir por uno mismo la solución. En realidad, la parte más dificil de ia resolucion de un problema matemático es, muy a menudo, "empezar", "ponerse en marcha", "conectar". $Y$ es facil subestimar las cualidades tanto de determinación como de imaginación que pueden necesitarse para ello.

Ayudar a la solidificación de aspectos psicológicos que propician el aprendizaje:

interes por cl trabajo que se hace
interés en el propio progreso
significatividad de la tarea
atencion reflexiva
atusencia de emoción no pertinente
pérdida de miedo al ridiculo
eliminacion de los mecanismos de defensa
que cualquiera tiene ante una situación
nueva, en particular un problema.

Fomentar la actitud básica en matemáticas: la de resolver problemas.

Situar a todos los alumnos en igual de condiciones al comienzo de una tarea.

Cuando se propone en clase calcular la ecuación de la cangente a la curva $y=\sqrt{\operatorname{tg}} x$ en el punto de ella que tiene como abscisa $\pi / 3$, se establece indefectiblemen(e una particion que rompe la clase en tres bloques: el de los que tienen los conocimientos técnicos adecuados y la necesaria confianza en sí mismos; el de los que saben que desconocen las herramientas imprescindibles 
y quedan automáticamente fuera de juego; y un tercer grupo intermedio que probablemente se verá frustado a! final por algún error conceptual o àgún fallo de memoria.

De acuerdo en que la mayor parte del trabajo mate. mático se hace sobre elementos técnicos que hay que tener adquiridos. Y en que no puede permanecerse en el bachillerato en un campo de problemas que no re. quieran conocimientos previos. Pero la importancia de la adquisición de herramientas técnicas no deberia hacer olvidar que la gran limitación que habitualmente rodea las matemáticas escolares y medias es la de proponer Irabajos cerrados, de respuesta única y ya respondidos por otros. Explorar, formular preguntas, conjeturar, reorganizar las propias conjeturas, producir generalizaciones, son los rasgos que constituyen la parte más atractiva de la actividad matemática y los que hacen que las matemáticas puedan ser de utilidad.

El pensamiento reproductivo, es decir, el hábito de usar respuestas aprendidas tiene dos ventajas: es más fácil de ensenar y es más fácil de someter a exámenes. El pensamiento productivo, que consiste en crear nuevas soluciones y usar nuevas organizaciones, es más dificil de enseñar, requiere más tiempo y necesita de otros esquemas de temporalización de la ensentanza y de estructuración de la clase.

Anthos constituyen los polos del conflicto entre el principio de inercia y el principio de acción en la ensentanza. Lo que sigue, contado a través de dos historias, quisre sugerir algunas razones para inclinarse a favor del segundo principio.

\section{PRIMERA HISTORIA}

Octubre. Tres dias de trabajo. Principales personajes: 38 atumnos de primer curso de bachillerato. Comienza asi:

Profesor.- «En este cuadro

\begin{tabular}{|c|c|c|c|}
\hline 16 & 3 & 2 & \\
\hline 5 & 10 & 11 & 8 \\
\hdashline & 6 & 7 & \\
\hline 4 & 15 & 14 & 1 \\
\hline
\end{tabular}

faltan los números 9,12 y 13 .

¿Donde crees que deben colocarse?"

Siguen unos pocos minutos de silencio y actividad. Se levantan varias manos. El profesor acude a ver lo que quieren decir. "Aquí, aquí y aqui, porque así todas las filas suman lo mismo, 34». Orras manos, "Aqui, aqui y aquí, porque veo que todas las filas y todas las columnas suman lo mismo." Más manos.

Profesor. "Quienes han puesto aqui e! 9 y aqui el 12, ¿por qué no los han puesto en el otro orden?"
Poro despues: "Ya lo tengo. Es mejor cambiarlos porque asi..."

El profesor escribe en la pizarra la colocación que la gran mayoria considera adecuada y pregunta "iHay alguien que haya encontrado la solucion de otra manera, es decir, sin sumas?»

Una alumna: "Si. Yo los he puesto de ese modo porque he visto que habia unas figuras
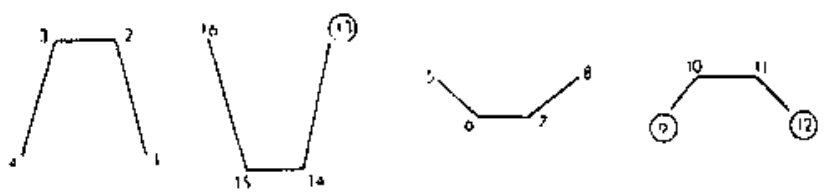

Profesor.- «Bien.* Ahora, en lugar de recibir el cua. dro hecho $\rightarrow$ casi hecho- vais a producir vosotros vuestros propios cuadros. Este es el problema:

"Busca un cuadro de tres filas y tres columnas tal que, estando formado por numeros todos distintos, la suma de cada fila, cada columna y cada diagonal sea la misma.

Seria preferible que no encontrases la solución por casualidad. sino que buscases un método, un procedimiento que te sirviese para más de una ocasión."

Al cabo de pocos minutos casi todos tienen escrito uno de esos cuadrados mágicos. El profesor pide para el dia siguiente que traigan escrita un cuadrado mágico que 'contenga en él infinitos a la vez, es decir que no tenga escritos solamente numeros sino letras que indiquen algo general*

Muchos llegaron sin tener más que una solución particular. Otros tenian varias. otros tenian un método, pero no sabian hacerlo explicito. Hubo quien renia la siguiente escritura 'general'.

\begin{tabular}{|c|c|c|}
\hline I & $F$ & $D$ \\
\hline$H$ & $G$ & $C$ \\
\hline$B$ & E & A \\
\hline
\end{tabular}

P.-

el ce

pensando, sin duda, que generalizar es poner letras sin dar leyes. Otro escribe en la pizarra

\footnotetext{
- El profesor habla esperado que este seria el procedimicnto elegido por la mayoría. ¿La razon? Que ese fue el procedimiento que èl siguió cuando vio ese cuadrado en "La melancolian, de Albert Durero.
} 


\begin{tabular}{|c|c|c|}
\hline $2 n$ & $-n$ & $2 n$ \\
\hline$-2 n+1$ & $2 n+1$ & $2 n+1$ \\
\hline $2 n$ & $2 n+1$ & $2 n$ \\
\hline
\end{tabular}

Profesor. $\rightarrow$ "Pon un ejemplon. Y tápidamente escribe

\begin{tabular}{|c|c|c|}
\hline 8 & -1 & 2 \\
\hline-3 & 3 & 9 \\
\hline 4 & 7 & -2 \\
\hline
\end{tabular}

confundiendo así dos numeros pares que, en su cabe$z a$, son distintos, con dos números pares que en la escritura son el mismo. Y revelando ese dato básico para la enseñanza que consiste en que la cantidad de conocimientos de que cada uno de nosotros es conscienle es muy inferior a la cantidad de lo que sabemos, y que la cantidad de conocimientos de los que somos conscientes es muy superior a la cantidad de conocimientos que sabemos verbalizar.

Preguntada la clase cómo se sale de tal situación, aigunos señalan que la escritura 'general' es incorrecta. Profesor. - «iAlguien tiene alguna que sea correcta?» Tres alumnos escriben cosas como esta:

\begin{tabular}{|c|c|c|}
\hline$A+1$ & $A-2$ & $A+3$ \\
\hline$A+4$ & $A$ & $A-4$ \\
\hline$A \cdot 3$ & $A+2$ & $A+1$ \\
\hline
\end{tabular}

P. - "Yo puedo escribir otras. Voy a empezar desde el centro, poniendo $a$ y luego poco a poco y con cuidado iré ajusıando lo que sea conveniente, asi:

\begin{tabular}{|c|c|c|}
\hline$a-1$ & $a-4$ & $a+5$ \\
\hline$a+6$ & $a$ & $a-6$ \\
\hline$a-5$ & $a+4$ & $a-1$ \\
\hline
\end{tabular}

$Y$ hora podéts hacer vosotros otras más generales, manteniendo la $a$, pero quitando importancia a los números que la rodean."

Casi en seguida alguien escribe en la pizarra

\begin{tabular}{|c|c|c|}
\hline$a-z$ & $a-4 z$ & $a+5 z$ \\
\hline$a+6 z$ & $a$ & $a+6 z$ \\
\hline$a-5 z$ & $a+4 z$ & $a+z$ \\
\hline
\end{tabular}

ENSEÑANZA DE LAS CIENCIAS
P. - «La clase ha terminado. El Irabajo que propongo para que traigáis manana es: a elegir entre escribir cuadrados mágicos $3 \times 3$ aún más generales o escribir cuadrados mágicos $4 \times 4$."

A la mañana siguente traen algunas cosas interesantes:

\begin{tabular}{|c|c|c|}
\hline$a-z$ & $a+z-y$ & $a+y$ \\
\hline$a+z+y$ & $z$ & $a-z-y$ \\
\hline$z-y$ & $a \cdot z+y$ & $a+z$ \\
\hline
\end{tabular}

\begin{tabular}{|c|c|c|c|}
\hline$a+1$ & $3+14$ & $a+15$ & $a+4$ \\
\hline$a+12$ & $a+7$ & $a+6$ & $a+9$ \\
\hline$a+8$ & $a+11$ & $a+10$ & $a+5$ \\
\hline$a+13$ & $a+2$ & $a+3$ & $a+16$ \\
\hline
\end{tabular}

y comentamos ambas:

En el de $4 \times 4$, "icómo lo has hecho», "Muy fácil, the mirado el primero que hicimos."

En el de $3 \times 3$, «Estupendo, aqui tenemos tres posibilidades en lugar de dos. ¿Cómo to has hecho?"; "He partido del cuadro [i] y donde pone 1 he puesto $z$, y donde pone $S$ he puesto y."; "Pon un ejemplo»; "Elijo, a capricho, $a=5, y=3, z=0$, con lo cual queda

\begin{tabular}{|c|c|c|}
\hline 5 & 2 & 8 \\
\hline 8 & 5 & 2 \\
\hline 2 & 8 & 5 \\
\hline
\end{tabular}

P.- «iPero eso no es un cuadrado mágico! No pueden elegirse a capricho a, z, y. Es preciso imponer condiciones. Mejor será que lo vuelvas a mirar en casa con atención.»

En efecto, lo miró con más atención e impuso las siguientes condiciones: "y es cualquier numero; $z$ es impar; $\mathbf{a}=z \mathbf{y}$.

Esa decisión de no darse por vencidos podria ser lo más destacable de esta historia si no hubiese habido algo aún más reconfortante: el hecho de que afrontason las diversas etapas que fueron presentándose sin buscar desesperadamente en su memoria y sin pedir con ansiedad la solución a otra persona.

\section{SEGUNDA HISTORIA}

Un día de diciembre. Personajes: 30 alumnos de segundo curso de bachillerato. En esta historia no hay conversaciones, sino escritura. El hilo de la trama fue el siguiente:

"44 es un numero feliz porque

$$
44 \rightarrow 4 x^{2}+42=32 \rightarrow 3^{2}+22^{2}=13 \rightarrow 1^{2}+3^{2}=10 \rightarrow 2^{2}+0^{2}=\text { (1) }
$$

Investiga sobre número felices."

El trabajo habia de hacerse en casa, sin limitación de tiempo, pero entregando por escrito el producto de la investigación. 
Se observará que el enunciado tiene un carácter ambiguo: ho se dice, por ejemplo, cuantas cifras ha de tener un número feliz; más aủn, no se da una definición precisa de lo que es un número feliz. Pero ¿es siempre conveniente eliminar toda ambigüedad en la presentacion de un problema? La polivalencia es una caracteristica generalmente consustancial a las situaciones $r i$ cas en contenido. Es del estudio de esas situaciones de donde debe venir la supresión de la ambigüedad, si ello es posible, precisando las condiciones iniciales y lievando a la consideración de las diversas opciones.

De acuerdo con ello, los personajes han interpretado con libertad. No todos los papeles pueden traerse aquí, pero incluiremos algunos significativos.

\section{Alfredo.}

Los numeros felices son aquellos en que la sucesiva suma de sus cuadrados da 1 .

Por ejemplo:

$44 \Rightarrow 4^{2}+4^{2}=32 \Rightarrow 3^{2}+2^{2}=13 \Rightarrow 1^{2}+3^{2}=$ $=10 \Rightarrow 1^{2}+0^{2}=1$.

Son numeros felices:

el 44 , el 23 , el 32 , el 31 , el 13, el 10 y la unidad seguida de ceros. Todos los numeros que contengan las anteriores cifras y que las restantes sean ceros:

44000; 40004; 400400; etc.

Todos los numeros que al sumr sus cuadrados nos dé la unidad seguida de ceros.

\section{Cosme}

(") Todas las potencias de 10 son "numeros felices". $10^{\prime \prime} \rightarrow 1^{2}+0^{2}+0^{2}+\ldots+0^{2}=1$

$2^{\circ}$ ) Si tenemos un "número feliz" de dos cifras, y las permutamos o invertimos sus posiciones, obtenemos olro "numero feliz».

$$
\begin{aligned}
& 13 \rightarrow 1^{2}+3^{2}=1+9=10 \rightarrow 1^{2}+0^{2}=1 \\
& 31 \rightarrow 3^{2}+1^{2}=9+1=10 \rightarrow 1^{2}+0^{2}=1 \\
& 32 \rightarrow 3^{2}+2^{2}=9+2=13 \rightarrow 1^{2}+3^{2}=10 \rightarrow 1^{2}+0^{2} \\
& =1 \\
& 23 \rightarrow 2^{2}+3^{2}=4+9=13 \rightarrow 1^{2}+3^{2}=10 \rightarrow 1^{2}+0^{2} \\
& =1
\end{aligned}
$$

3") Un "numero feliz» multiplicado por 10 o por potencias de 10 se transforma en otro "número feliz»: 13. $10^{n} \rightarrow 1^{2}+3^{2}+0^{2}+\ldots 0^{2}=1+9=10 \Rightarrow 1^{2}+0^{2}$ $=1$

\section{Antonio $\mathrm{A}$.}

E! 44 es un numero feliz porque

$44 \rightarrow 4^{2}+4^{2}=32 \rightarrow 3^{2}+2^{2}=13 \rightarrow 1^{2}+3^{2}=10 \rightarrow$ $1^{2}+0^{2}=1$

La definición de un numero feliz es cuando la suma de los cuadrados de las componentes es (1)

Números felices:

- Todos los números que estén compuestos por la unidad seguida de ceros. Ej.:

$1000 \rightarrow 1^{2}+0^{2}+0^{2}+0^{2}=1$
- El cuadrado de 3 más el cuadrado de I da 10, por lo que todos los numeros que la suma de sus cuadra. dos da 13031 , o estos mismos numeros son números felices.

$31 \rightarrow 3^{2}+1^{2}=10 \rightarrow 1^{2}+0^{2}=1$

$13 \rightarrow 1^{2}+3^{2}=10 \rightarrow 1^{2}+0^{2}=1$

- El cuadrado de 8 más el cuadrado de 6 da 100 , por lo que todos los numeros que la suma de sus cuadrados da 68 o 86. o estos mismos números son números felices.

$68 \rightarrow 6^{2}+8^{2}=100 \rightarrow 1^{2}+0^{2}+0^{2}=1$

$86+8^{2}+6^{2}=100 \rightarrow 1^{2}+0^{2}+0^{2}=1$

Conclusion:

Vamos a tomar como números felices todos los números cuya suma de los cuadrados de las componentes de los siguientes números: $31,13,68,86$. También dichos numeros seguidos de ceros, 0 que los ceros vayan entre medias de las dos cifras.

Emilio.

- Los números felices del I al $100 \mathrm{son}$

$1,7,10,13,19,23,28,31,32,44,49,68,70,79,82,86,9 !$, $94,97,100$.

- Todos los demás van dando sumas parciales que se repiten constantemente y que siempre acaban en el $\mathrm{N}^{\circ}$ Ejemplo:

is $\Rightarrow 1^{2}+5^{2}=1+25=26 \Rightarrow 2^{2}+6^{2}=4+36$ $=40 \Rightarrow 4^{2}=16 \Rightarrow 1^{2}+6^{2}=1+36=37 \Rightarrow$ $3^{2}+7^{2}=9+49=58 \Rightarrow 5^{2}+8^{2}=25+64=897$ $8^{2}+9:=64+81=145 \Rightarrow 1^{2}+4 i+5^{2}=1+16+$ $25=42 \Rightarrow 4:+2:=16+4=20 \Rightarrow 2^{2}=4$

$66 \Rightarrow 6^{2}+6^{2}=36+36=72 \Rightarrow 7^{2}+2^{2}=49+$ $4=53 \Rightarrow 5^{2}+3^{2}=25+9=34 \Rightarrow 3^{2}+4^{2}=9+$ $+16=25 \Rightarrow 2^{2}+5^{2}=4+25=29 \Rightarrow 2^{2}+9^{2}=$ $4+81=85 \Rightarrow 8^{2}+5^{2}=64+25=89 \Rightarrow$ $8^{2}+9^{2}=64+81=145 \Rightarrow 1^{2}+4^{2}+5^{2}=1+16+$ $25=42 \Rightarrow 4^{2}+2^{2}=16+4=20 \Rightarrow 2^{2}+0^{2}=4$ - Por tanto, podemos decir que si en un $n^{\circ}$ cualquiera efectuamos las primera sumas y no nos da alguno de los numeros felices del 1 al 100 , es que no es feliz ejem. $1582 \Rightarrow 1^{2}+5^{2}+8^{2}+2^{2}=1+25+64+4$ $=94$

si que es feliz, luego 1582 es feliz.

$$
2327 \Rightarrow 2^{2}+3^{2}+2^{2}+7^{2}=4+9+4+49=66
$$

no es feliz, luego 2327 tampoco lo es.

\section{Antonio B.}

El 44 es un número feliz.

A un número de dos o más cifras se le considera feliz cuando la suma del cuadrado de cada una de sus cifras da un numero al ctual aplicamos la misma formula $y$ así sucesivamente hasta que de el número final $\rfloor$. 
Como cjemplo más sencillo diremos que el 10, 100. 1000 etc. son numeros felices yá que la suma del cuadrado de sus cifras es 1 por eso diremos como primera ley que todo número que sea un I seguido de los ceros que se quiera es un numero feliz.

Deducimos asi que numeros felices hay infinitos pero no sólo de multiplos de 10 elevado a h sino yue con esto deducimos una segunda ley que será que cualquier numero que de como suma del cuadrado de sus cifras un numero que sea un 1 seguido de ceros, scrá numero feliz.

Ejem.

$94111 \Rightarrow 9^{2}+4^{2}+1^{2}+1^{2}+1^{2}=100 \Rightarrow 1^{2}+0^{2}+0^{2}=1$ $564331 \Rightarrow 5^{2}+6^{2}+4^{2}+3^{2}+3^{2}+1^{2}=100 \Rightarrow 1^{2}+0^{2}+0^{2}$ $=1$

$103 \Rightarrow 1^{2}+0^{2}+3^{2}=10 \Rightarrow 1^{2}+0^{2}=1$

$99999986 \Rightarrow 9^{2}+9^{2}+9^{2}+9^{2}+9^{2}+8^{2}+6^{2}=1000 \Rightarrow$ $\mathrm{1}^{2}+0^{2}+0^{2}+0^{2}=1$

Numero super feliz es:

y9999999999999999999999 y 100 nueves más y' seguidamente $61 \Rightarrow 9^{2}+y^{*}$ etc. ......... $=10000 \Rightarrow 1$

Si se atresc y tiene tiempo comprucbelo.

Ora conclusion a la que podemos llegar es que es también numero feliz aquel al cual aplicando la fórmula acostumbrada nos da un numern que a su ver nos da un numero que sea $10^{\mathrm{n}}$ y asi seguidaments

Eicm. 622 $\Rightarrow 44 \Rightarrow 32 \Rightarrow 13 \Rightarrow 10 \Rightarrow 1$

Y como ultima conclusión diré que para sacar más numeros felices podemos cambiar y revolver el orden de las cifras ya que hay un dicho que dice " $E$ l orden de los sumandos no altera la suman.

Es lo mismo poner 94111 que $104119041911 \mathrm{etc}$ Si nos ponemos en este plan no dejamos de hacer números felices hasta el dia del juicio final

$9+11 ! \Rightarrow 100 \Rightarrow 1$

$104119 \Rightarrow 100 \Rightarrow 1$

$41911 \Rightarrow 100 \Rightarrow$ !

Y terminando con los números felices yo dire que tambièn soy teliz porque mi cuadrado da 1 y porque por fin lo he sacado.

La lectura de los 30 trabajos permite observar una gradación de dificultades $-y$ de exitos- en la investigación:

- La etapa empírica (ver si tal y tal otro número son o no felices).

Buena parte de los alumnos no pasa de esta etapa.

- El descubrimiento de leyes generales: ceros intercalados, permutaciones de las cifras,...

Pero este descubrimiento es solo una parte dentro de la investigacion porque hay que advertir la necesidad de considerar todos los casos, o dicho de otro modo, encontrar
- La manera de generar números felices. El halliago de un método: 'comenzar desde el final' como forma de salir de lo empírico (a partir del i llego al 10. del 10 llego al 130 al 31 -y al 310 y al $103 \ldots$ - del 103 llego al...); (a partir del 1 llego al 100 o al $1000 \ldots$ )

- El sacar partido de circunstancias fortuitas: el hecho imprevisto y chocante - senalado solmaente por un alumno- de que si no se llega al I se llega al 4. (No estaria bien ignorar que este alumno, Emilio, tenia un rendimiento 'académico' por debajo de la media.)

- La escritura final de los resultados. En la que se advierte en no pocos casos una destreza completamente inhabitual en las situaciones que hemos llamado de pensamiento reproductivo.

Quizás le agrade al lector participar como un personaje más en una tercera historia. Basta con que dedique un poco de su tiempo (algunos alumnos de $3^{\circ}$ han dedicado mucho) a este problema:

“En un papel cuadriculado se loma un rectángulo con sus vértices situados en puntos del reticulo.

Se traza una diagonal.

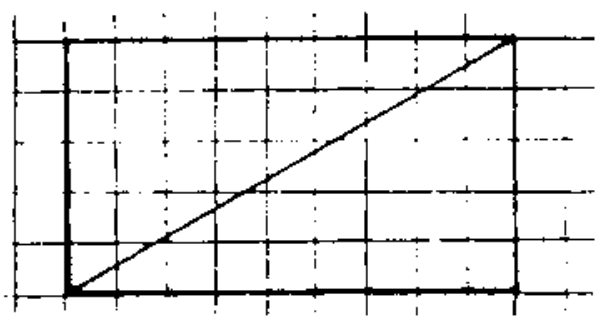

¿A cuántos cuadrados unidad corta esa diagonal?

Generaliza. Demuestra.»

Seguramente tendrá ocasión de comprobar que gran parte de las dificultades y de las recompensas quie halie al resolverlo serán muy semejantes a las que lienen los alumnos.

No exagera Dienes cuando afirma que la ensetama de las matemáticas ta! como suele hacerse no mejora nin. guna parte de la mente, excepto posiblemente tia memoria, porque ni la inventiva, ni la investigatión. ni la imaginación están presentes en su aprendizaje habitual.

De ahi que el fracaso escolar no es solo el de los que suspenden los exámenes, sino también el de los que los aprueban. $Y$ yo no veo que se pueda despegar hacia el éxito más que desde dos puntos de partida que se apoyan mútuamente: I) las matemáticas son un modo de conocer y no un modo de observar to que orros co. nocen; II) lo que pueda haber de estimulante en la labor de un profesor proviene menos de su pasión por enseñar que de su pasión por aprender, menos de su habilidad para impartir conocimientos que de su capacidad para subordinar éstos a la actrvidod cognoscitivo de sus alumnos. 
Bellas palabras liene Caleb Gattegno ("Mathematics Teaching', $n^{\circ} 100$. Septiembre de 1982) para convocar a esa tarea:

«EL futuro exigirá más y más implicación en el es. tudio del aprendizaje por parte de todos aquellos que aprenden (entre los cuales se incluyen los profeso- res), de modo que la ensetanza pueda ser desarrollada como la ayuda que las viejas generaciones ofrezcan a las más jovenes con objeto de favorecer la evolución humana sobre la tierra. No hay ningún otro significado para la educación. Hacer cualquier otra cosa es perder el tiempo.

¿Deseas tú, querido lector, hacer realmento eso?» 